\title{
Acknowledgement to Reviewers of Buildings in 2016
}

\section{Buildings Editorial Office,}

Published: 12 January 2017

MDPI AG, St. Alban-Anlage 66, 4052 Basel, Switzerland; buildings@mdpi.com

The editors of Buildings would like to express their sincere gratitude to the following reviewers for assessing manuscripts in 2016.

We greatly appreciate the contribution of expert reviewers, which is crucial to the journal's editorial process. We aim to recognize reviewer contributions through several mechanisms, of which the annual publication of reviewer names is one. Reviewers receive a voucher entitling them to a discount on their next MDPI publication and can download a certificate of recognition directly from our submission system. Additionally, reviewers can sign up to the service Publons (https://publons.com) to receive recognition. Of course, in these intiatives we are careful not to compromise reviewer confidentiality. Many reviewers see their work as a voluntary and often unseen part of their role as researchers. We are grateful to the time reviewers donate to our journals and the contribution they make.

If you are interested in becoming a reviewer for Buildings, see the link at the bottom of the webpage http://www.mdpi.com/reviewers.

The following reviewed for Buildings in 2016:

Adekunle, Timothy O.

Amin, Mohamad Hassan

Anranter, Michael

Arcuri, Natale

Asdrubali, Francesco

Balaras, Constantinos A.

Bard, Delphine

Bastos, Joana

Belayachi, Naima

Belgiu, Mariana

Belleri, Andrea

Bellia, Laura

Bignon, Jean-Claude

Billah, Muntasir

Biserni, Cesare

Bokel, Regina

Bordallo, Heloisa N.

Bouldin, John C.

Bourdais, Romain

Bournet, Pierre-emmanuel

Brischke, Christian

Buccolieri, Riccardo

Capener, Carl-Magnus

Caselles, Vicente

Cavalline, Tara

Cetin, Kristen S.
Chan, Ying-Chieh

Chatzidiakou, Lia

Chen, Bing

Chen, Chi-Feng

Chen, Jiayu

Chen, Zhiyong

Cheze, David

Chiesa, Giacomo

Clerc, Laurent

Conceição, Eusébio Z.E.

Dabaieh, Marwa

D'Agostino, Delia

De Risi, Raffaele

De Stefano, Mario

Derrible, Sybil

Dewsbury, Mark

Diamanti, Maria Vittoria

Domingo-Irigoyen, Silvia

Ergan, Semiha

Falkenbach, Heidi

Fayazbakhsh, Mohammad Ali

Fazenda, Bruno

Fernandes, Luis

Fernández-Solís, José L.

Fioretti, Roberto

Galvão, João Rafael 
Garcia, Maria Da Luz

Gentile, Niko

Gheisari, Masoud

Ghosh, Aritra

Gilani, Sara

Girhammar, Ulf Arne

Golasi, Iacopo

Gorgolis, Georgios

Grobman, Yasha Jacob

Guichard, Stéphane

Han, Yilong

Hartmann, Timo

Hatzigeorgiou, George D.

Hengstberger, Florian

Henriques, Fernando M.A.

Henry, Michael

Hernández-Pérez, Iván

Hoes, Pieter-Jan

Hoffmann, Sabine

Hong, Tianzhen

Honjo, Tsuyoshi

$\mathrm{Hu}$, Jia

Huang, Hao

Hughes, Ben Richard

Hunt, Dexter V.L.

Hwang, Chao-Lung

Ibrahim, Mohamad

Ignatius, Marcel

Iyer-Raniga, Usha

Jeon, Jin Yong

Jimenez Delgado, Antonio

Jung, Young-Soo

Junnila, Seppo

Kantsa, Aphrodite

Karol, Elizabeth

Kim, Jae Ik

Kim, Sun Sook

Koller, Alexander

Kovacic, Iva

Kramer, Rick

Le, Anh Dung Tran

Leonforte, Fabrizio

Levermore, Geoff

Lim, Jae-Han

Lokki, Tapio

Lollini, Roberto

Loonen, Roel

Macchioni, Nicola

Macdougall, Colin

Madonna, Francesco

Maeda, Akira

Magli, Susanna
Marinakis, Vangelis

Marique, Anne-Françoise

Mark, Ole

Marsh, Rob

Marshall, Maria I.

Martinelli, Paolo

Martin-Gomez, Cesar

Maskulk, Md

Mateus, Nuno

Matson, Eric T.

Mauro, Gerardo Maria

Mavromatidis, Lazaros

Mayo, Glenda

McCarty, Chris

McGrattan, Kevin B.

McNeil, Andrew

Meng, Lexuan

Messina, Francesco

Messineo, Antonio

Mirzaei, Parham

Mistrick, Richard G.

Mollazadeh, Mohammad H.

Montero, Jorge Isaac Peren

Moobela, Cletus

Mora, Rodrigo

Motamedi, Ali

Muhammad-Sukki, Firdaus

Muscio, Alberto

Mwasha, Abrahams

Nakanishi, Shinsuke

Nocera, Francesco

Noori, Mehdi

Noro, Marco

O’Brien, William

Oldfield, Philip

Oliveira Panao, Marta J.N.

Osello, Anna

Pachego-Torgal, Fernando

Palme, Massimo

Papadimitriou, Christina N.

Park, Kenneth

Parys, Wout

Paul, Christopher

Peffer, Therese

Pereira, Luisa Dias

Pienaar, Josua

Pinho, Luís

Piselli, Cristina

Pisello, Anna Laura

Pons, Oriol

Porras Alvarez, Santiago

Pour Rahimian, Farzad 
Powell, Michael

Pozza, Luca

Prasad, Prasad

Pretlove, Stephen

Pretot, Sylvie

Pusceddu, Cristina

Pyo, Sukhoon

Razmara, Meysam

Richard, Roger Bruno

Ryan, Chris

Sainz, Beatriz

Sajjadian, Masoud

Salata, Ferdinando

Salvalai, Graziano

Saneinejad, Saba

Schwartz, Yair

Scioscia, Floriano

Sciurpi, Fabio

Scorpio, Michelangelo

Secchi, Simone

Sharifi, Ayyoob

Shea, Andy

Shimizu, Takafumi

Shyr, Oliver F.

Silva, Cristina Matos

Silvestre, Jose Dinis

Soleimani-Mohseni, Mohsen

Sprecher, Aaron

Stafford, Anne

Stephenson, Max O.

Suarez, Rafael
Sun, Yongjun

Taleghani, Mohammad

Tarabieh, Khaled

Tasinari, Patrizia

Theodosiou, Theodoros

Trivess, Moore

Tsai, Huan-Liang

Ullrich, Torsten

Vale, Brenda

Van De Kuilen, Jan Willem

Vermeir, Gerrit

Vernardou, Dimitra

Versini, Pierre-Antoine

Waldmann, Daniele

Walker, Peter

Wang, Hai

Wang, Wei-Chih

Wehrden, Henrik Von

Williams, Joe

Woodbury, Rob

Wootton-Beard, Peter

Xuan, Jin

Yang, Jiachuan

Yin, Rongxin

Yuan, Jihui

Yun, Jieheerah

Zach, Jiří

Zalejska-Jonsson, Agnieszka

Zea Escamilla, Edwin

Zhao, Xianbo

(c) 2017 by the author; licensee MDPI, Basel, Switzerland. This article is an open access article distributed under the terms and conditions of the Creative Commons Attribution (CC-BY) license (http://creativecommons.org/licenses/by/4.0/). 